\section{Notes \& News}

At the meeting of the Psychonomic Society last fall, the Governing Board voted to authorize this Journal to carry Notes \& News of events of interest to members of the Society as well as to its readers. This is the first column devoted to such news. You are encouraged to send us any newsworthy item concerning change of position, promotion, fellowships, or special events. Because some readers subscribe to only one section, we will duplicate items in the two sections so that all of you will "get the word."

The first Paul M. Fitts Memorial Lectures were presented at the University of Michigan on March 25 through April 3 by Donald E. Broadbent, Director of the Medical Research Council Applied Psychology Research Unit, Cambridge, England. The general topic of the lectures was Decision and Stress. They are an updating of Dr. Broadbent's book on Perception and Communication, and will probably be published as a book in the next year or two. Dr. Broadbent was recently elected to Fellowship in the Royal Society.

A new publication, the Journal of Motor Behavior, is scheduled to appear in March, 1969. Members of its Editorial Board include Arthur Irion, Clyde Noble, and Don Trumbo; its Managing Editor is Richard A. Schmidt of the University of Maryland.

Wilse B. Webb, retiring as chairman of the Department of Psychology, University of Florida in July, 1969, will go to Cambridge, England in September on a National Science Foundation Faculty Development Award. Irwin Silverman of the University of Buffalo and Clifford H. Swensen, Jr., of Purdue University have been spending a sabbatical year at the University of Florida during the 1968-1969 academic year.

Daniel P. Kimble has been awarded an NSF Science Faculty Postdoctoral Fellowship for a sabbatical year (1969-1970) at the Institute of Experimental Psychology, University of Oxford, Oxford, England. He has also been promoted to the rank of Professor at the University of Oregon.

Abram Amsel of the University of Toronto will join the faculty of the University of Texas in August, 1969, as Professor of Psychology.

\title{
Auditory differential conditioning of the rabbit nictitating membrane response: I. Effects of mixed-and separate-phase training'
}

PATRICK O'MALLEY, RALPH B.
HUPKA, and JOHNW.MOORE, University
ofMassachusetts, Amherst, Mass. 01002

This study compared several procedures for establishing differential conditioning with tonal stimuli. Eight albino rabbits received 3 days of differential conditioning consisting daily of 60 reinforced $(\mathrm{CS}+)$ trials randomly interspersed with unreinforced (CS-) trials (mixed-phase or D). After each of 32 rabbits received 60 trials of continuous reinforcement (CRF) on Day 1, Ss were divided into four groups (separate phase): (a) Group CRF-D was given 60 differential conditioning trials for the remainder of that session; (b) Group CRF-I-D received 10 $C S$ - trials and completed the session on Day 1 with 50 differential conditioning trials; (c) Group 2 CRF-2I-D (10-20-10) received $10 C S-, 20 C S+, 10 C S-$, and 20 differential trials; and (d) Group 2 CRF$2 I-D$ (5-10-5) was given $5 C S-, 10 C S+$, $5 C S-$, and 40 differential conditioning trials. All separate-phase Ss received 120 trials/day of conventional differential conditioning on Days 2 and 3. The separate-phase Ss demonstrated a superior rate of differentiation on Day 2 in comparison with the mixed-phase Ss, but by Day 3 the latter Ss had reached a similar rate of differentiation.

The purpose of this experiment was to compare various training schedules for establishing differential conditioning. A conventional mixed-phase procedure, in which the reinforced (CSt) and nonreinforced (CS-) conditioned stimuli are presented equally often according to a random sequence, was contrasted with various separate-phase procedures in which differentiation followed an initial series of $\mathrm{CS}+$ trials. There were four variations of this separate-phase procedure in all, three involving a long series of $\mathrm{CS}-$ trials following the initial acquisition series. On the second and third day of training all groups were compared on a mixed-phase schedule.

According to a simple conditioningextinction theory of discrimination learning, one in which excitation and inhibition combine algebraically and in which sequential or contrast effects are minimized, the rate of differentiation and the level of responding to each $\mathrm{CS}$ of a particular pair depends only on the number of CSt and CS- trials and not on their pattern of presentation. Number of trials is presumably the only essential variable in determining the growth of excitation and inhibition to the two CSs, and hence the amount of generalization of these processes between CS+ and CS-. Such a model has proved adequate when applied to human eyelid conditioning experiments employing either separate- or mixed-phase schedules (Gynther, 1957;Homzie, 1968).

\section{SUBJECTS}

The Ss were 40 naive, male and female New Zealand White rabbits, 90-120 days old.

\section{APPARATUS}

Four rabbits were run concurrently in a four-drawer fire-proof filing cabinet. Each drawer was serviced by separate and independent control and recording equipment located in an adjoining room. A panel in front of each $\mathrm{S}$ supported two house lights ( $4 \mathrm{~V} \mathrm{dc}$ behind translucent white plastic) and three impedance matched speakers which provided tones and a continuous white masking noise of $75 \mathrm{~dB}$ SPL. Each drawer was independently ventilated by centrifugal blowers. The animals were restrained in clear plastic boxes identical to those developed by Gormezano (1966). The superior and inferior external eyelids of S's right eye were kept open by means of small clips. The hook-up to the nictitating membrane was accomplished by means of a minitorque potentiometer and surgical suture arrangement similar to that described by Gormezano. Movements of the right nictitating membrane were amplified and recorded on a Grass $5 \mathrm{D}$ inkwriting oscillograph. A conditioned response (CR) was defined as any $1-\mathrm{mm}$ positive deflection of the recording pen within the interstimulus interval (ISI). The UCS consisted of a 2-mA ac shock of 50-msec duration applied to wound-clip electrodes on the infraorbital region of the right eye. Duration of the CS was equal to the ISI plus $50 \mathrm{msec}$. The pure tones used as CSs were generated by Hewlett-Packard audio-oscillators 